\title{
Commentary to paper "Primary versus secondary delayed gastric emptying (DGE) grades B and C of the International Study Group of Pancreatic Surgery after pancreatoduodenectomy: a retrospective analysis on a group of 132 patients"
}

\author{
Alessandro Zerbi ${ }^{1} \cdot$ Giovanni Capretti $^{1}$
}

Received: 4 August 2015/Accepted: 10 August 2015/Published online: 28 August 2015

(C) Italian Society of Surgery (SIC) 2015

Delayed gastric emptying (DGE) is usually considered the most frequent complication of pancreatoduodenectomy (PD), after pancreatic fistula and its septic and hemorrhagic sequels. In the past, the incidence of DGE was reported as highly relevant, approaching 40-50\% [1]. The efforts, mainly by the International Study Group on Pancreatic Fistula (ISGPS), in careful recording and classifying complications after PD, lead to a better knowledge of rate and characteristics of this complication. In particular, the severity of DGE has been precisely defined, similarly to other complications, like pancreatic fistula: type A DGE, clinically not relevant, was clearly separated from grade $B$ and $\mathrm{C}$. After the introduction of this classification, nearly all papers adopted this terminology, making much more easy to compare different reports in terms of DGE occurrence: we now usually refer to grade $B$ and $C$ of DGE in evaluating postoperative course of PD.

The pathogenesis of DGE has always represented an important topic of debate, and also in the present paper by Courvoisier et al. [2] it is widely discussed. Several reasons have been proposed, like disruption of the vagal nerve system, ischemic injury to the antro-pyloric mechanism, decreased plasma motilin stimulation due to resection of duodenum, transient torsion or angulation of the reconstructed alimentary tract. However, none of these mechanisms has been convincingly demonstrated to be the main reason for DGE occurrence after PD.

Alessandro Zerbi

alessandro.zerbi@humanitas.it

1 Pancreatic Surgey Section, Humanitas Research Hospital, Via Manzoni 56, 20089 Rozzano, MI, Italy
Many efforts aimed to reduce the incidence of DGE have been attempted: pyloric dilatation, preservation of right gastric artery, preoperative use of erythromycin; none of these proved to be effective. Also the preservation of the pylorus was suggested to be related to the occurrence of DGE, and some authors speculated on the role of the length of the preserved portion of duodenum at this regard. However, also the decision to preserve the pylorus instead to perform an antrectomy, as in the classical Whipple procedure, seemed to not influence the occurrence of DGE, as underlined by a recent metanalysis [3].

More relevant from a clinical point of view is the observation that DGE is most often secondary to the presence of a pancreatic fistula and its related complications, and very seldom a primary event [1]. Also in the present paper by Courvoisier et al. [2], this observation is underlined and supported by convincing data. Only in less than one-third of cases DGE is a primary event and occurs as an isolated complication: clinical relevance of DGE after PD is then less important than it could appear from its rough rate of incidence, because in secondary DGE the clinical picture is overlooked by pancreatic fistula and its sequels. Moreover, if we consider that the incidence of DGE after PD has decreased during the years (grade B-C DGE is now currently reported in $10-15 \%$ of cases), we understand that the role of this complication in the postoperative course of PD is less relevant than in the past. There are two main reasons for the decrease in DGE incidence: the widespread use of antecolic reconstruction of the gastro/duodenojejunostomy and the introduction of ERAS protocols.

For what antecolic reconstruction is concerned, after evidence provided by a randomized study from Japan [4] of a sharp decrease in the rate of DGE when antecolic 
reconstruction for gastric continuity after PD is chosen, this technical choice gained widespread diffusion. The distance between the stomach and the pancreatic stump when antecolic reconstruction is chosen, probably protects the stomach form effusions at the pancreatic anastomotic site, thus avoiding possible impairments in stomach motility.

A further decrease in DGE occurrence was observed in association with the introduction of ERAS protocols. The immediate removal of nasogastric tube (at the end of intervention) and the early reassumption of liquid (on the first postoperative day) and solid food (on 3th-4th postoperative day), allow an early recovery of gastrointestinal function. Together with early mobilization, administration of prokinetics drugs, careful patient education, this policy proved to obtain a faster recovery after PD without increasing postoperative complications [5].

In conclusion, DGE after PD provides an interesting example of how, after a careful classification and understanding of complications, the introduction of technical surgical variations and the modification of postoperative management can significantly affect the occurrence of specific postoperative complications.
Compliance with ethical standards

Conflict of interest None.

\section{References}

1. Zhou Y, Lin J, Wu L, Li B, Li H (2015) Effect of antecolic or retrocolic reconstruction of the gastro/duodenojejunostomy on delayed gastric emptying after pancreaticoduodenectomy: a metaanalysis. BMC Gastroenterol 15:68

2. Courvoisier T, Donatini G, Faure JP, Danion J, Carretier M, Richer JP (2015) Primary versus secondary delayed gastric emptying (DGE) grades $\mathrm{B}$ and $\mathrm{C}$ of the International Study Group of Pancreatic Surgery after pancreatoduodenectomy: a retrospective analysis on a group of 132 patients. Updates Surg. doi:10.1007/ s13304-015-0296-1

3. Diener MK, Knaebel HP, Heukaufer C et al (2007) A systematic review and meta-analysis of pylorus-preserving versus classical pancreaticoduodenectomy for surgical treatment of periampullary and pancreatic carcinoma. Ann Surg 245:187-200

4. Tani M, Terasawa H, Kawai $M$ et al (2006) Improvement of delayed gastric emptying in pylorus-preserving pancreaticoduodenectomy: results of a prospective, randomized, controlled trial. Ann Surg 243:316-320

5. Chaudhary A, Barreto SG, Talole SD et al (2015) Early discharge after pancreatoduodenectomy: what helps and what prevents? Pancreas 44:273-278 